\title{
Transcriptional activation by estrogen receptor (ER $\alpha)$ and steroid receptor coactivator (SRC1) involves distinct mechanisms in yeast and mammalian cells
}

\author{
H M Sheppard, S Matsuda, J C Harries, K B Kindle and D M Heery \\ Department of Biochemistry, University of Leicester, University Road, Leicester LE1 7RH, UK \\ (Requests for offprints should be addressed to D M Heery; Email: dh37@le.ac.uk)
}

\begin{abstract}
Steroid receptors activate transcription in yeast cells via interactions with endogenous coactivators and/or basal factors. We examined the effects of mutations in the ligand binding domain on the transcriptional activity of ERo in yeast. Our results show that mutations in Helix 3 (K366A) and Helix 12 (M547A, L548A) disrupt transcriptional activity of $\mathrm{ER} \alpha$ in yeast, as previously observed in mammalian cells. However, replacement of a conserved tyrosine residue in Helix 12 with alanine or aspartate (Y541A and Y541D), which renders $E R \alpha$ constitutively active in mammalian cells, had only a weak stimulatory effect on ligand-independent reporter activation by $E R \alpha$ in yeast. Two-hybrid interaction experiments revealed that a Y541A mutant expressed in yeast was capable of ligand-independent binding to a mammalian coactivator, suggesting that there is a subtle difference in how this mutant interacts with mammalian and yeast cofactors. We also show that the ligand-dependent activities of ER $\alpha$ and progesterone receptor (PR) in yeast cells were strongly enhanced by the human p160 protein steroid receptor coactivator (SRC1), but not by CREB-Binding Protein (CBP) or the p300/CBP associated factor (P/CAF). Although the SRC1 activation domains $A D 1$ and $A D 2$ are functional in yeast, deletion of these sequences only partially impaired SRC1 coactivator function in this organism; this is in contrast to similar experiments in mammalian cells. Thus SRC1 sequences involved in recruitment of CBP/p300 and Co-ActivatorAssociated Arginine Methyltransferase (CARM-1) in mammalian cells are not essential for its function in yeast, suggesting that SRC1 operates via distinct mechanisms in yeast and mammalian cells.
\end{abstract}

Journal of Molecular Endocrinology (2003) 30, 411-422

\section{Introduction}

The molecular machinery involved in gene transcription is well conserved among the eukaryotes (Guarante \& Bermingham-McDonogh 1992). Yeast and mammalian cells share homologous RNA polymerases, basal factors, mediator proteins, chromatin remodelling factors and chromatin modifying enzymes. This has facilitated functional studies of mammalian transcription factors in the genetically tractable yeast organism. The nuclear receptors (including steroid, retinoid, vitamin D and thyroid receptors) activate reporter gene transcription in the presence of cognate ligands in yeast cells (McEwan 2001). The ligand binding domains (LBDS) of ERa (Pierrat et al. 1992) and progesterone receptor (PR) (Mak et al. 1989, Meyer et al. 1992) containing the $\mathrm{AF} 2$ transactivation function have been shown to mediate liganddependent reporter activation in yeast. The LBD of $\mathrm{ER} \alpha$ also contains a minor activation domain (AF2-a) that binds mammalian $\mathrm{TAF}_{\mathrm{II}} 30$ (Jacq et al. 1994). This sequence was originally identified as a transcriptionally active domain in yeast (Pierrat et al. 1994), although its molecular target in that organism is unknown. As in mammalian cells, the AF1 transactivation domain located within the $\mathrm{N}$-terminus of steroid and retinoid receptors is constitutively active in yeast cells when separated from the LBD (Pierrat et al. 1992, Metzger et al. 1988, 1992, 1995, Heery et al. 1993). Synergistic interactions between $\mathrm{AF} 1$ and $\mathrm{AF} 2$ domains have 
also been observed for retinoic acid receptor heterodimers in yeast (Heery et al. 1993, 1994, Hall et al., 1993, Allegretto et al. 1993). Mutations in Helix 3 and Helix 12 that disrupt ER $\alpha$ transcriptional activity (Danielian et al. 1992, Henttu et al. 1997) result in loss of coactivator recruitment via LXXLL motifs (Heery et al. 1997). Thus, nuclear receptor sequences necessary for cofactor recruitment in mammalian cells are also necessary for transcriptional activity in yeast cells.

Homologs of nuclear receptor coactivators such as p160s, PGC1 and TRAP220 appear to be absent from yeast, although other components of coactivator complexes are conserved in this organism e.g. GCN5, SRB/Med proteins. Similarly, CBP and p300 have no close sequence homologs in yeast, although they share conserved functional domains, such as the bromodomain and a histone acetyltransferase domain, with a number of yeast proteins. In addition, a cysteine/ histidine rich region of $\mathrm{CBP} / \mathrm{p} 300$ shows partial homology with the yeast coactivator ADA2p (Guarente \& Bermingham-McDonogh 1992). Genetic screens for yeast proteins required for the function of steroid receptors have identified HSP90 (Picard et al. 1990), SWI2/SWI3 proteins (Yoshinaga et al. 1992, Wallberg et al. 2000), GCN5, ADA proteins (vom Baur et al. 1998, Anafi et al. 2000) PSU1 (Gaudon et al. 1999), SPT6 (Baniahmad et al. 1995), RSP5 (Imhof et al. 1996) and several other cofactors (McEwan 2001). In this study we assess the effects of mutations in Helices 3 and 12 on the transcriptional activity of $\mathrm{ER} \alpha$ in yeast. We also compare the abilities of the mammalian coactivators $\mathrm{SRC1}, \mathrm{GBP}$ and $\mathrm{P} / \mathrm{CAF}$ to enhance the activities of steroid receptors in yeast, and define sequences in SRG1 responsible for its activity in this organism.

\section{Materials and methods}

\section{Plasmid constructs}

The reporter p3ERE-lacZ (also termed pRL $\Delta 21 \mathrm{U} 3$ ERE), consisting of three estrogen response elements within a minimal URA3 promoter flanking the E.coli lacZ gene, has been described previously (Metzger et al. 1995). cDNAs encoding full length SRC1e, SRC1a (Kalkhoven et al. 1998), CBP (Sheppard et al. 2001) and P/CAF (Yang et al. 1996) were subcloned into Yep20 (TRP1 selectable) or Yep30 (HIS3 selectable) vectors, which are modified versions of Yep10 and Yep90, respectively (Heery et al. 1993, 1994) containing multiple cloning sites within a $P G K$ promoter cassette and a consensus Kozak's sequence. The mouse $\mathrm{ER} \alpha$ wild type and mutant cDNAs were cloned into the Yep90 (HIS3 selectable) vector. The construct DBD-PR consists of the progesterone receptor LBD (amino acids 633-933) fused in frame with the human ER $\alpha$ DNA binding domain (DBD) in the vector pBL1 (HIS3 selectable) (Le Douarin et al. 1995, 2001). The SRC1 deletion mutant series was generated by PCR, introducing flanking restriction sites, and a Kozak's sequence and initiation codon at the 5' end of each PCR fragment, before subcloning into the Yep20 vector. SRC1 mutants containing the $\triangle \mathrm{AD} 1$ deletion (amino acids 901-950) were PGR amplified using the pSG5hSRC1e $\Delta$ ADl construct as a template (Sheppard et al. 2001). The DBD-SRC1 series used to map the transactivation domains of SRC1 in yeast were also generated by PCR and subcloned in frame with the DBD in the pBL1 vector. All PGR constructs were fully sequenced.

\section{Yeast reporter assays}

Yeast W303 or BY4704 cells were cotransformed with p3ERE-lacz reporter and cDNA expression vectors encoding wild type or mutant ER $\alpha$. For coactivator activity assays, yeast cells containing the reporter were cotransformed with expression plasmids encoding ER $\alpha$ or DBD-PR in combination with various coactivators expression plasmids, or empty vector controls, and maintained on selective media. For yeast two-hybrid assays, yeast cells were cotransformed with Yep90 ER $\alpha$ and pASV3 (VP16) (Le Douarin et al. 2001) or pASV3-mouse SUG1 (VP16-SUG1) (vom Baur et al. 1996). Co-transformants were grown in liquid culture for $16 \mathrm{~h}$ in the presence of $10^{-6} \mathrm{M}$ 17- $\beta$-estradiol (E2), progesterone (R5020) or vehicle (ethanol) as required. For mapping the activation domains of SRC1, yeast cells were cotransformed with reporter and the DBD-SRC1 series or empty vector pBL1 controls. Reporter $\beta$-galactosidase activity in yeast cell-free extracts (prepared by the glass bead method) was measured quantitatively as described previously (Le Douarin et al. 2001). In experiments where cell growth arrest was induced by ligands, cells were cultured to late 
log phase prior to addition of the ligand, and reporter activity was assessed after a further $8 \mathrm{~h}$. Reporter activity is expressed as units of specific activity (nmoles/mg/min).

\section{Western blots}

Yeast cells expressing recombinant proteins were cultured to late log phase in a selective liquid medium $(15 \mathrm{ml})$. Cell pellets were resuspended in a buffer containing $1 \mathrm{M}$ sucrose, $25 \mathrm{mM}$ EDTA, $50 \mathrm{mM}$ dithiothreitol (in a final volume of $1 \mathrm{ml}$ ). Sphaeroplasts were prepared by incubating $100 \mu \mathrm{l}$ of the cell slurry with 1 unit Zymolyase 20T (Seikagaku America, Falmouth, MA, USA) for $1 \mathrm{~h}$ at $37^{\circ} \mathrm{C}$. Sphaeroplasts were harvested by low speed centrifugation, resuspended in sample buffer, boiled for $10 \mathrm{~min}$, and aliquots of the cell-free extracts were separated on $6 \%$ or $12 \%$ acrylamide gels by SDS-PAGE, followed by transfer to nitrocellulose. For transfer of larger proteins such as $\mathrm{CBP}$ to nitrocellulose, high glycine buffer (300 mM) was used. Western blotting was carried out using standard procedures. Recombinant ER $\alpha$ expressed in yeast was detected using a 1:5000 dilution of a monoclonal antibody raised against human ER $\alpha$ (anti-F region mouse monoclonal, a gift from $\mathrm{P}$ Chambon) which cross-reacts with mouse ER $\alpha$. Controls for the western blot include cell-free extract from yeast expressing human ER $\alpha$ (1-595) and transiently transfected COS-1 cells over-expressing mouse $\operatorname{ER} \alpha(1-599)$ (shown in Fig. 1F), or SRCle (Fig. 2C). Polyclonal antisera purchased from Santa Cruz Biotechnology Inc. (Santa Cruz, CA, USA) were as follows; SRC1 C20 (sc-6096), SRC1 M20 (sc-6098), CBP A22 (sc-369), CBP C20 (sc-583) and P/CAF (sc-6300). The polyclonal antibodies were used at a dilution of 1:500. For detection of CBP, best results were obtained using both anti-CBP antibodies in combination. Peroxidase-linked anti-goat, antimouse or anti-rabbit secondary antibodies were used to detect the proteins using ECL Plus detection kits (Amersham, Buckinghamshire, UK).

\section{Results}

\section{Point mutations in Helix 3 and Helix 12 of the LBD disrupt the function of ER $\alpha$ in yeast}

Previous studies revealed that mutation of amino acids such as L543, L544, M547or L548 in Helix 12, or K366 in Helix 3, leads to loss of cofactor binding in vitro and reduced transcriptional activity by mouse $\mathrm{ER} \alpha$ in mammalian cells (Danielian et al. 1992). In contrast, replacement of Y541 in Helix 12 with alanine or acidic residues did not perturb ligand-dependent activity, but resulted in a strong ligand-independent activation of $\mathrm{ER} \alpha$ (White et al.1997). These amino acids are comprised within the LBD AF2 surface that accommodates recruitment of coactivators via LXXLL amphipathic $\alpha$-helices (Heery et al. 1997). The importance of key residues in Helix 3 and Helix 12 for coactivator binding has been highlighted by the structure of the LBD of PPAR $\gamma$ in complex with a portion of the SRG1 nuclear receptor interaction domain (Nolte et al. 1998), and the $\mathrm{ER} \alpha \mathrm{LBD}$ complexed with LXXLL peptides (Shiau et al. 1998). The amino acids K366 and E542, which are highly conserved throughout the nuclear receptor family, appear to form a charged clamp that locks the LXXLL $\alpha$-helix into the AF2 channel (Nolte et al. 1998).

To characterise the effects of similar mutations on the transcriptional activity of ER $\alpha$ in yeast cells, reporter activation by $\mathrm{ER} \alpha$ mutants was assessed. The domain structure and relative positions of amino acids mutated in this study are depicted in Fig. 1A. As shown in Fig. 1B, mutation of the conserved hydrophobic residues M547 and L548 to alanine resulted in almost complete loss of the ligand-dependent activity of $\mathrm{ER} \alpha$, as previously observed in mammalian cells (Danileian et al. 1992). Consistent with this, we have previously shown that a VP16-ER $\alpha$ construct containing these mutations failed to bind to LXXLL peptides or full-length coactivators in yeast cells (Heery et al. 1997). Similarly, mutation of K366 to alanine resulted in a substantial loss of ligand-dependent activity of $\mathrm{ER} \alpha$, as demonstrated by comparison of the ligand dose-dependent reporter activation of $\mathrm{ER} \alpha$ with that of $\mathrm{ER} \alpha \mathrm{K} 366 \mathrm{~A}$ (Fig. 1C). These results indicate that the AF2 surface is important for transcription in both mammalian and yeast cells, and suggests that yeast proteins mediating ER $\alpha$ activity may contact $\mathrm{AF} 2$ in a similar fashion to mammalian coactivators.

Our experiments revealed that ligand-dependent reporter activation by the $\mathrm{ER} \alpha$ mutants $\mathrm{Y} 451 \mathrm{D}$, Y541E and Y541F was very similar to that observed for wild type $\mathrm{ER} \alpha$, although we noted a reproducible $1 \cdot 7$-fold increased level of reporter activation by Y541A, compared to wild type or other Y541 mutants (Fig. 1D). Reporter activation 
A Mouse ER $\alpha$

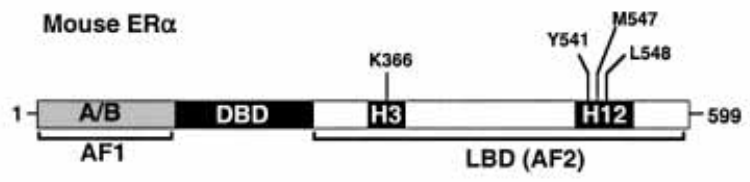

B

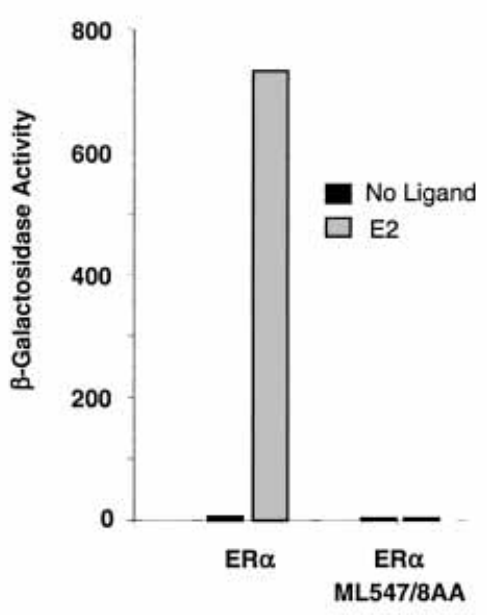

D

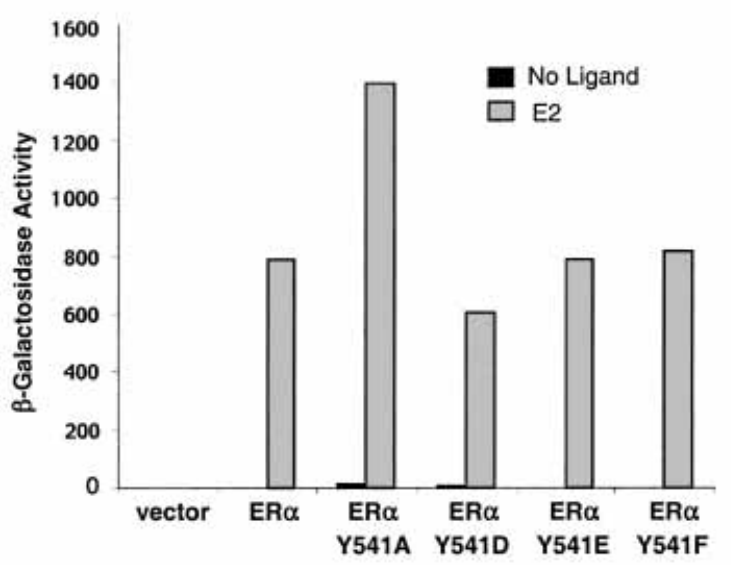

$\mathrm{F}$

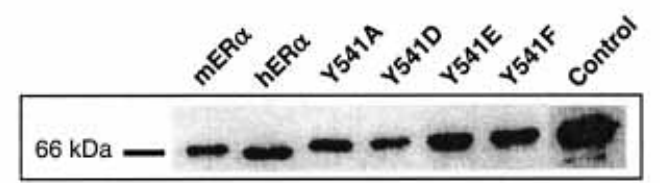

C

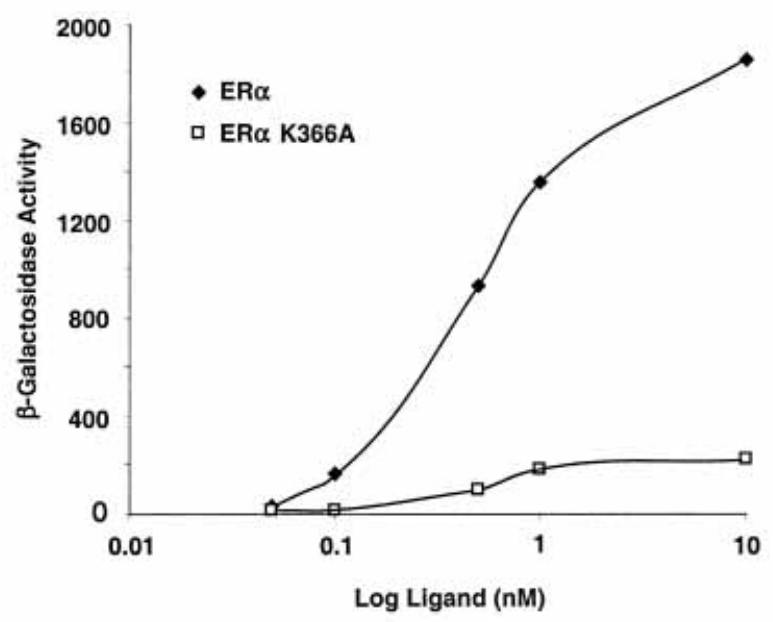

E
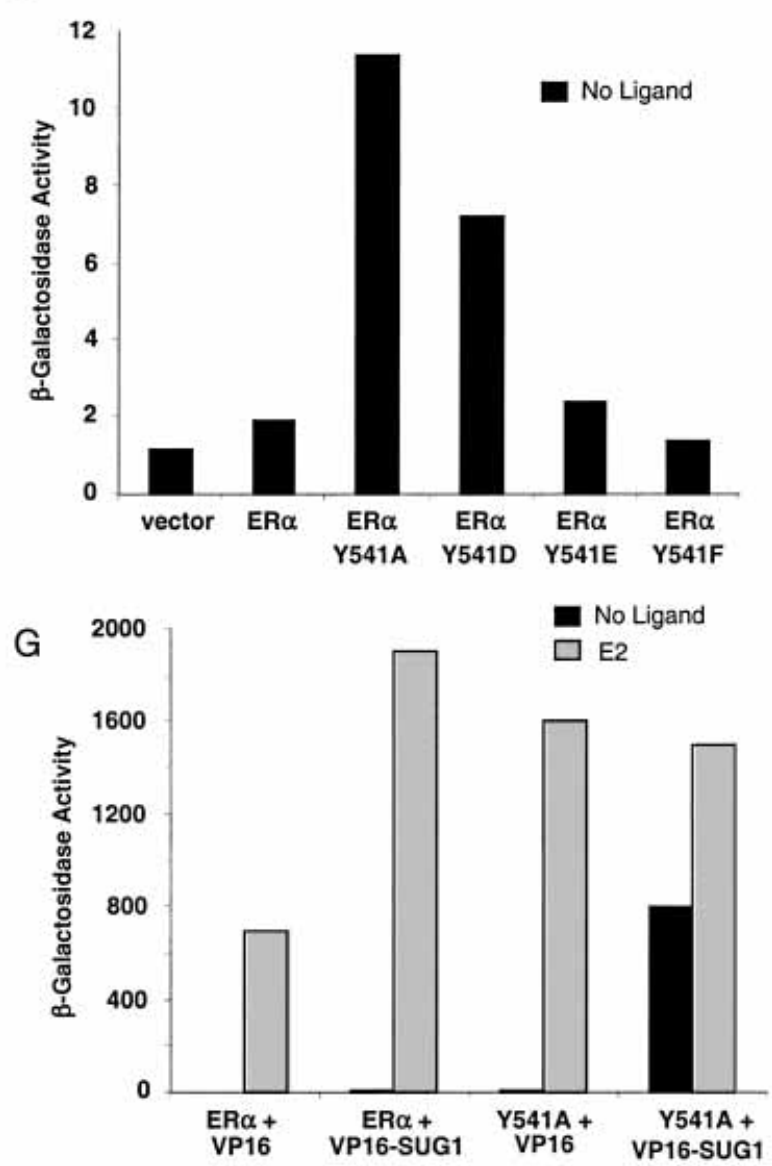
by Y541A and Y541D in the absence of ligand was 6-fold and 3.5 fold greater, respectively, than that observed for wild type $\mathrm{ER} \alpha$ whereas Y541E and Y $541 \mathrm{~F}$ showed no substantial increase in ligandindependent reporter activation (Fig. 1E). The expression of wild type and mutant proteins at similar levels was confirmed by western blotting (Fig. 1F). Thus, in contrast to similar assays in mammalian cells (White et al. 1997), Y541 mutants do not have strong constitutive transcriptional activity in yeast cells, but rather show a weak increase in ligand-independent reporter activation. To examine whether the Y541 mutants retained their ability to interact with a mammalian coactivator in a constitutive manner, we performed yeast two-hybrid assays. Whereas the interaction of wild type ER $\alpha$ with VP16-SUG1 was almost entirely dependent on ligand, the Y541A mutant showed a very high level of reporter activation (800-fold over basal) when coexpressed with VP16-SUG1 in the absence of ligand (Fig. 1G), indicating constitutive interaction of these proteins in yeast. The mouse SUG1 protein has been shown to bind $\mathrm{ER} \alpha$ and other $\mathrm{NRs}_{\mathrm{s}}$ via $\mathrm{AF} 2$ (Lee et al. 1995, vom Baur et al. 1996). Thus, while the Y541A mutation stimulates ligand-independent interaction with a mammalian coactivator in yeast, it does not result in constitutive activity of the mutant ER $\alpha$, suggesting that recruitment of yeast cofactors is not substantially affected by this mutation. Our results imply a subtle difference in the way yeast and mammalian cofactors interact with ER $\alpha$ LBD.

\section{SRC1 proteins, but not CBP or P/CAF, enhance steroid receptor activity in yeast}

cDNAs encoding human SRCla, human SRGle, human P/CAF and mouse CBP (depicted schematically in Fig. 2A) were cloned into the yeast expression vector Yep20. The yeast strain W303 (or BY4705) was cotransformed with plasmids p3ERE-lacZ (Metzger et al. 1995) and Yep90 mouse ER $\alpha$. Transformants containing these plasmids were then further transformed with Yep20 empty vector, or Yep20 containing a cDNA encoding a mammalian coactivator. Reporter activity was assessed after overnight liquid culture in the presence or absence of E2, as outlined in Materials and methods. As shown in Fig. 2B, yeast cells expressing ER $\alpha$ alone showed a strong induction of reporter activity in the presence of ligand, whereas negligible reporter activity was observed in its absence. Co-expression of $\mathrm{ER} \alpha$ with $\mathrm{P} / \mathrm{CAF}$ or $\mathrm{CBP}$ did not substantially enhance or decrease the reporter activity, either with or without ligand (Fig. 2B). However coexpression of human SRCila enhanced the reporter activity approximately 4 -fold in the presence of ligand. In addition, a strong increase in ligand-independent activity was observed, although this level of reporter activity remained 10 -fold lower than that induced by E2 (Fig. 2B). Similar ligandindependent enhancement of $\operatorname{ER} \alpha$ activity by SRC1 isoforms has been observed in transiently transfected mammalian cells (Sheppard et al. 2001, White et al. 1997, Kalkhoven et al. 1998 Bevan et al. 1999). Interestingly, coexpression of the human SRCle isoform with $\mathrm{ER} \alpha$ strongly enhanced the ligand-independent reporter activity, but had only a modest effect on the ligand-induced activity (Fig. 2B). Analysis of cell morphology in transformants co-expressing ER $\alpha$ and SRGle in the presence of ligand revealed that the majority of cells were atypical elongated cells or pseudohyphal structures (Fig. 2E). Similar phenotypes were previously observed in cells undergoing growth arrest in liquid media, due to expression of VP16-ER $\alpha$ fusion

Figure 1 (A) Schematic representation of the mouse $E R \alpha$ showing domain structure including the $N$-terminal $(A / B)$, DNA binding (DBD) and ligand binding (LBD) domains. The regions associated with transactivation functions (AF1 and $A F 2$ ) are indicated. The regions encoding Helices 3 and 12 and residues mutated in this study are also shown. (B) Reporter $\beta$-galactosidase activity in cell-free extracts from yeast cells expressing mouse $E R \alpha$ wild type, or $E R \alpha$ ML547/8AA as indicated, in the presence or absence of ligand. (C) Reporter activity measured in cell-free extracts of yeast cells expressing $\mathrm{ER} \alpha$ wild type, or $\mathrm{ER} \alpha \mathrm{K} 366 \mathrm{~A}$ in the presence of various concentrations of ligand as indicated. (D) Reporter activity in cell-free extracts of yeast cells expressing ER $\alpha$ wild type, or ER $\alpha$ Y541 mutants in the presence or absence of ligand. (E) Ligand-independent reporter activities as shown in (D) using a different unit scale. (F) Western blot showing expression of mouse and human $E R \alpha$, and mouse $E R \alpha$ mutants expressed in yeast cells. The control consists of extract of transiently transfected COS-1 cells expressing the mouse ER $\alpha$. (G) Yeast two-hybrid experiment, showing reporter activites in cell-free extracts of yeast cells co-expressing wild type ER $\alpha$ or the ER $\alpha$ Y541A with VP16 or VP16-SUG1 in the presence or absence of ligand as indicated. 
A
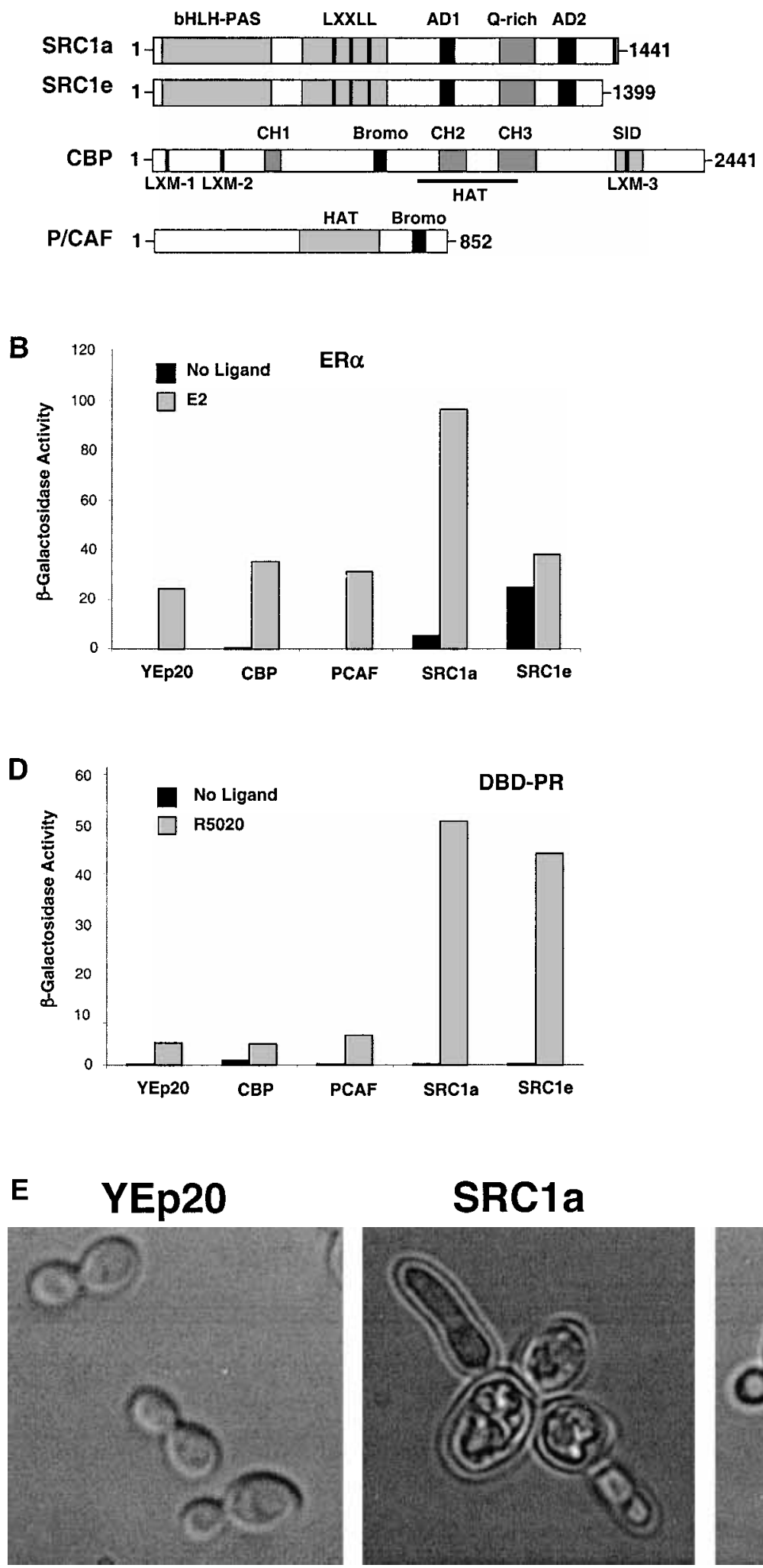

SRC1a
C

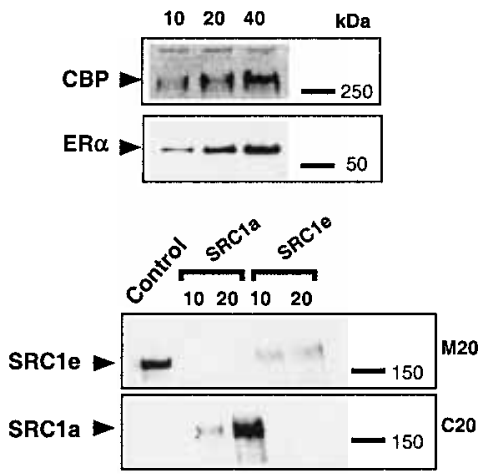


proteins in the presence of ligand (Gilbert et al. 1993). Cells expressing SRCila were also found to have atypical cell growth in the presence of ligand, albeit to a lesser extent, and similar results were obtained in both the W303 and BY4705 strains (data not shown). Thus, reporter activation by $\mathrm{ER} \alpha / \mathrm{SRC} 1$ proteins (in particular SRC1e) in the presence of ligand is likely to be underestimated in these clones due to deleterious effects on cell growth by transcriptional interference or 'squelching'. No squelching effects were observed in yeast cells expressing $\mathrm{ER} \alpha$ with $\mathrm{CBP}$ or $\mathrm{P} / \mathrm{CAF}$, or due to expression of $\mathrm{SRCl}$ proteins in the absence of $\mathrm{ER} \alpha$ (data not shown).

The expression of full length $\mathrm{ER} \alpha, \mathrm{CBP}$ and SRC1 proteins in yeast cell free extracts was confirmed by western blotting (Fig. 2C). However, we were unable to detect expression of full-length $\mathrm{P} / \mathrm{CAF}$ in yeast extracts using a commercially available anti-P/CAF antibody, although we did detect expression of LexA-tagged $\mathrm{P} / \mathrm{CAF}$ constructs using an anti-LexA antibody, and these proteins also failed to enhance $\mathrm{ER} \alpha$ activity (data not shown).

In previous studies we reported that, in mammalian cells, the ligand-independent effect of $\mathrm{SRCl}$ on $\mathrm{ER} \alpha$ is mediated by the interaction of the $\mathrm{AF} 1$ domain of $\mathrm{ER} \alpha$ with the glutamine-rich (Q-rich) domain of SRC1 (Sheppard et al. 2001, Bevan et al. 1999). To test whether this was also the case in yeast, we transformed BY4705 with a construct consisting of the LBD of the progesterone receptor fused to the DBD of the $\mathrm{ER} \alpha$ (DBD-PR). The steroid receptors $\mathrm{ER} \alpha$ and $\mathrm{PR}$ show no differences in their interactions with p160s, $\mathrm{CBP} / \mathrm{p} 300$ or $\mathrm{P} / \mathrm{CAF}$ in reporter assays in mammalian cells, or in vitro. As shown in Fig. 2D,
DBD-PR gave a moderate but reproducible enhancement of reporter activity in yeast in the presence of the agonist ligand R5020. This level of reporter activation is approximately 4-5-fold lower than ER $\alpha$ on the same reporter (Fig. 2B) or full length PR on a PRE-driven reporter gene (data not shown). This reflects the loss of synergistic interaction with the $\mathrm{AF} 1$ domain, as reported previously for other steroid receptors (Pierrat et al. 1992, Heery et al. 1993). As observed with full length $\mathrm{ER} \alpha$, the co-expression of $\mathrm{P} / \mathrm{CAF}$ or $\mathrm{CBP}$ proteins did not have a substantial effect on the ligand-dependent or ligand-independent reporter activities. However, a strong stimulation of ligand-dependent reporter activity was observed in cotransformants expressing either SRCla or SRCle (Fig. 2D). In contrast to the full length $\mathrm{ER} \alpha$, no ligand-independent activity was observed with DBD-PR, due to the absence of the AF1 domain. In addition, the overall reporter activity saturates at a lower level, and we did not observe squelching effects using the DBD-PR construct.

\section{Sequences required for SRC1 coactivator activity in yeast}

To determine which domains of SRG1 are required for its ability to enhance transcriptional activity of the PR AF2 in yeast, we constructed a series of SRC1 deletion mutants in the vector Yep20 (Fig. 3A). We then assayed reporter activity due to DBD-PR in cells co-expressing the SRC1 mutants. As shown in Fig. 3B, C-terminal truncations up to amino acid 980 increased the ligand-dependent reporter activity approximately 2-5-fold over wild type. This may result from an inhibitory effect of the C-terminus on SRC1

Figure 2 (A) Schematic representation showing domain structure of mammalian coactivators SRC1a, SRC1e, CBP and P/CAF. Functional domains indicated include the basic Helix-Loop-Helix (bHLH) and Per-Arndt-Sim (PAS) domains, LXXLL motifs (LXM1-3 in CBP), activation domains (AD1 and AD2), glutamine rich region (Q-rich), cysteine/histidine rich regions ( $\mathrm{CH} 1-3)$, SRC1 interaction domain (SID), bromodomain (bromo) and histone acetyltransferase (HAT) domain. (B) Reporter $\beta$-galactosidase activity in cell-free extracts from yeast cells co-expressing mouse ER $\alpha$ with mammalian coactivator proteins as indicated, in the presence or absence of ligand. (C) Western blot using specific antibodies to detect mammalian proteins in yeast cell-free extracts as indicated. The amount of cell-free extract loaded $(10,20$, or $40 \mu \mathrm{l})$ is indicated. The control for SRC1 western blots is cell-free extract of transiently transfected COS-1 cells over-expressing SRC1e. The antibodies M20 and C20 are specific for SRC1e and SRC1a, respectively. The approximate molecular weights are indicated. (D) Reporter $\beta$-galactosidase activity in cell-free extracts from yeast cells co-expressing DBD-PR with mammalian coactivator proteins as indicated, in the presence or absence of ligand. (E) Phase contrast images (magnification 100X) showing morphology of yeast cells co-transformed with Yep90 ER $\alpha$ and Yep20, Yep20 SRC1a or Yep20 SRC1e. 
A

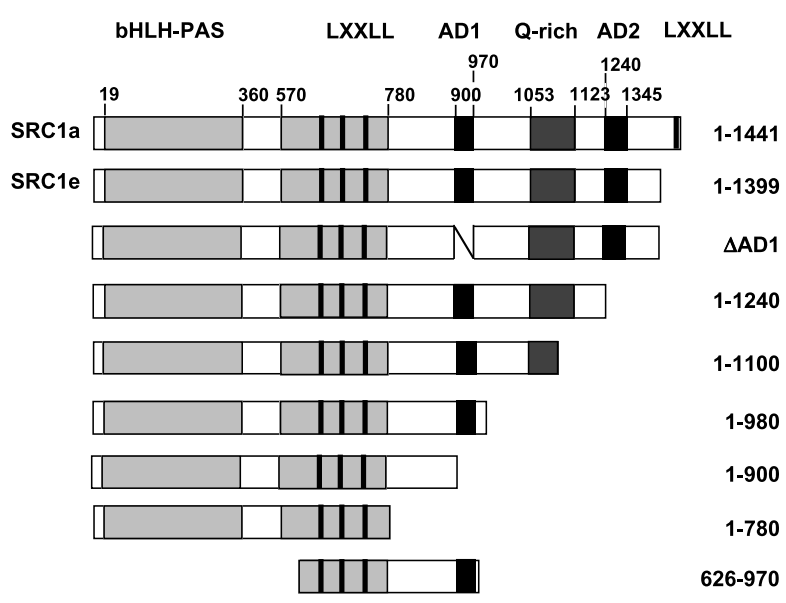

B

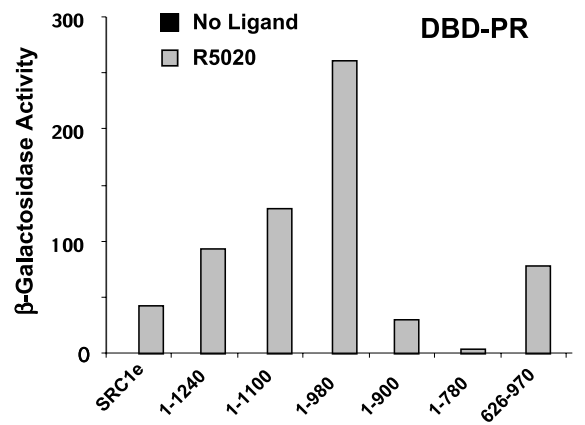

C

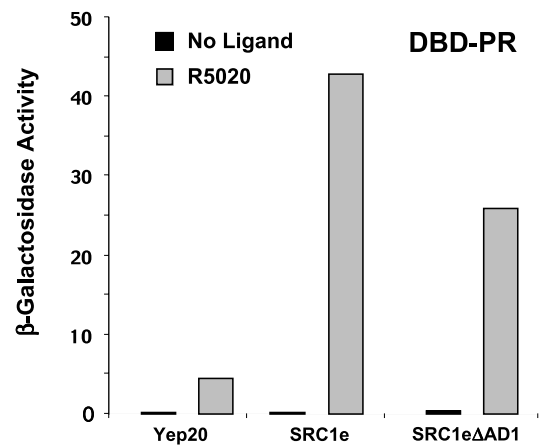

Figure 3 (A) Schematic representation of SRC1 constructs cloned in the Yep20 expression vector. Functional domains (as in Fig. 2) are indicated. (B) and (C) Effect of SRC1 constructs on DBD-PR mediated reporter activation in the presence or absence of ligand as indicated.

interaction with endogenous coactivators, or another unknown mechanism. Note, that while we did not observe the drastic cell growth arrest phenotype, we cannot rule out the possible squelching effects in cells expressing proteins containing the C-terminal domain of SRC1e. In any case, the highest activity was observed with SRC1 1-980, indicating that the AD2 domain and the Q-rich region are not critical for the ability of SRC1 to enhance PR activity in yeast. In contrast, however, truncation at amino acid 900, which deletes $\mathrm{AD} 1$, resulted in a decrease in the ligand-dependent reporter activity to a level lower than that achieved with full length SRG1. This indicates an involvement of the ADl domain in achieving the maximal activity, although the reporter activity was still markedly (8-10-fold) higher than that due to DBD-PR alone. The result contrasts with studies in mammalian cells indicating a requirement for the $\mathrm{AD} 1$ domain and $\mathrm{CBP}$ recruitment (Sheppard et al. 2001, Li et al. 2000, Kim et al. 2001). Further truncation of SRG1 up to amino acid 780, resulted in a dramatic reduction in activity to a level similar to that obtained in the absence of exogenous coactivator (Fig. 2D \& 3B). These results suggest that the sequence between 780 and 900, in addition to the $\mathrm{AD} 1$ region, may mediate interactions with yeast cofactors and thus contribute to SRG1 coactivator function in yeast.

To determine whether the bHLH-PAS domain and N-terminal sequences of SRG1 are important in yeast, we constructed the mutant SRC1 626-970, which retains only the central nuclear receptor interaction domain (NID) and the adjacent AD1 domains. As shown in Fig. 3B, this construct maintains a strong coactivator activity (almost 2-fold greater than full length SRC1e). A similar result was obtained in mammalian cells in which the minimal SRC1 construct was equally potent as the full length SRCle (Sheppard et al. 2001).

Deletion of the AD1 region (Helix A amino acids 901-950, see below) in the context of the full-length protein SRC1e (SRC1e- $\triangle \mathrm{AD} 1$ ) resulted in only a $40 \%$ reduction in the ability of SRC1 to enhance AF2 activity (Fig. 3C). A similar result was obtained on comparing 1-1100 and 1-1100 $\mathrm{ADD} 1$ (data not shown). Taken together, our data suggest that several surfaces on SRC1, including AD1 and the sequence between 780-900, mediate interactions with endogenous yeast cofactors resulting in enhanced ligand-dependent transcription by ER $\alpha$ or PR LBDs. 
A

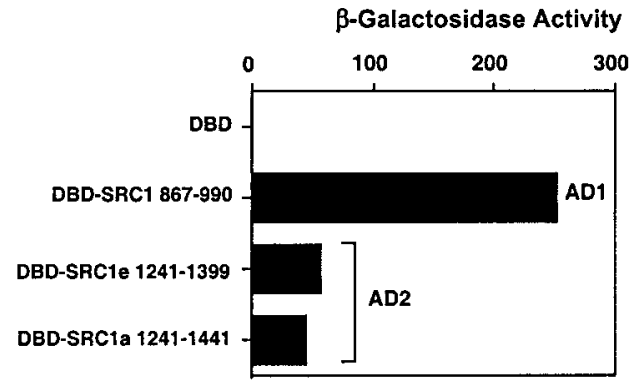

B
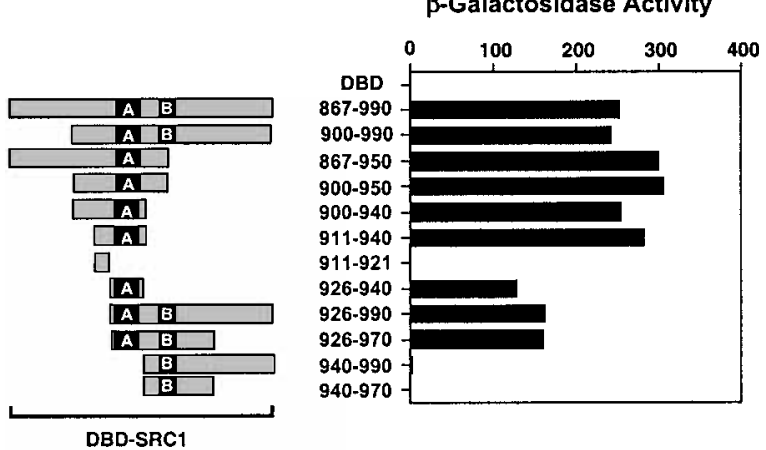

$\mathrm{C}$

926- RNDEKALLEQLVSFL -940

Helix-A

Figure 4 (A) Transcriptional activity of DBD-SRC1 constructs in yeast containing the AD1 and AD2 domains as indicated. (B) Deletion mapping of SRC1 $A D 1$ region to identify the sequence responsible for transcriptional activity. Helix $A$ and Helix $B$ sequences are denoted by the black boxes. (C) Sequence of the region encoding Helix $A$. The $\alpha$-helical region as defined in the CBP/ACTR interaction domain structure Demarest et al, 2002, is indicated.

\section{SRC1 activation domains (AD1 and AD2) are functional in yeast}

Two regions of SRCl have been shown to be involved in transcriptional activation of reporter genes in mammalian cells when fused to a heterologous DBD (Onate et al. 1995, Kalkhoven et al. 1998, Sheppard et al. 2001). These regions are AD1 (926-970), which acts via recruitment of the global co-activators $\mathrm{CBP}$ and p300, and AD2, which functions by binding the methyltransferases CARM1 and PRMT1 (Chen et al. 1999). As shown in Fig. 4A, a construct consisting of SRC1 amino acids 867-990 fused to the ER $\alpha$ DBD produced a very high level of reporter activity in yeast, indicating that the $\mathrm{ADl}$ region is also a potent transcriptional activation domain in this organism. Similarly, the C-terminal sequences from SRC1e (1241-1399) and SRG1a (1241-1441), which contain the AD2 domain, are also strong activators in yeast, albeit at a level of activity 5 fold lower than $\mathrm{AD} 1$. These results suggest that yeast cofactors bind to sequences within the 2 major activation domains of SRC1.

To map the sequence responsible for the transcriptional activity of SRC1 867-990 more precisely, we generated a series of DBD-SRC1 fusion proteins spanning the $\mathrm{AD} 1$ region. As shown in Fig. 4B, the minimal sequence having transcriptional activity was contained within amino acids 926-940 of SRC1. This region contains Helix A of the AD1 domain (Sheppard et al. 2001, Demarest et al. 2002), which is not sufficient on its own to bind CBP or activate transcription in mammalian cells (Sheppard et al. 2001). Thus, our results highlight a difference in the way SRC1 AD1 domain functions in yeast and mammalian cells, consistent with the absence of a sequence homolog of $\mathrm{CBP}$ in yeast.

\section{Discussion}

The structure of the human ER $\alpha$ LBD in complex with peptides derived from GRIP1 NR box 2 motif shows that K362, M543 and L544 (the human equivalents of mouse K366, M547 and L548) are involved in contacts with the GRIP1 LXXLL peptides (Shiau et al. 1998). In contrast, Y537 (the human equivalent of mouse Y541) does not appear to be in close contact with the LXXLL core motif sequence. However, sequences flanking the core motifs are known to be important for differential interactions of coactivators with steroid receptors and other NRs (Needham et al. 2000, Coulthard et al. 2003), although such sequences have yet to be observed in LBD/coactivator peptide crystal structures. Thus, it remains to be established how replacement of Y541 with alanine or acidic residues enhances ligand-independent binding of coactivators in mammalian cells. Our results show the Y541A mutation has only a very weak effect on the ligand-independent activity of $\mathrm{ER} \alpha$ in yeast (Fig.1D, Fig.1E). Nonetheless, we observed a strong ligand-independent interaction of $\mathrm{ER} \alpha \mathrm{Y} 541 \mathrm{~A}$ with the mammalian cofactor SUG1 in yeast cells (Fig. 
1G). A previous study has shown that SUG1 interaction with human $\mathrm{ER} \alpha$ requires Helix 12, and is also disrupted by mutations in M543 and L544, although similar mutations in Helix 12 of RAR $\alpha$ only partially disrupted SUG1 binding (vom Baur et al. 1996). Our results indicate that, in addition to increasing ligand-independent interaction with RIP140 and SRC1, the Y541A mutation also results in constitutive interaction with SUG1. However, our results also indicate a subtle difference in the way yeast and mammalian cofactors contact the LBD surface. Consistent with this, the yeast proteins known to interact with ectopically expressed nuclear receptors have not been demonstrated to contain functional LXXLL sequences.

Although CBP is required for the transcriptional activity of $\mathrm{ER} \alpha$ in mammalian cells and in vitro we have shown that direct interaction of full-length $\mathrm{CBP}$ with steroid and retinoid receptors is relatively weak, as the three LXXLL motifs present in CBP (LXM 1-3) have low affinity for steroid and retinoid receptors (Sheppard et al. 2001, Heery et al. 2001). Consistent with this, we report here that we did not detect any enhancement of $\mathrm{ER} \alpha$ activity due to co-expression with $\mathrm{CBP}$ in yeast. The $\mathrm{CBP} / \mathrm{p} 300$ associated factor $\mathrm{P} / \mathrm{CAF}$, which is related to the yeast GCN5 histone acetyltransferase, has been reported to interact weakly with the DBD of steroid and retinoid receptors in GST pulldown experiments, but without a requirement for ligand (Blanco et al. 1998). However, P/CAF did not enhance the activity of ER $\alpha$ or PR AF2 in our yeast reporter assays. Previous studies have shown that $\mathrm{P} / \mathrm{CAF}$ and $\mathrm{CBP}$ sequences can enhance transcription of reporter genes when tethered to promoters in yeast, suggesting they are at least partly functional in this organism. Therefore the failure of $\mathrm{P} / \mathrm{CAF}$ and $\mathrm{CBP}$ to enhance steroid receptor activity in our experiments may indicate a lack of interaction with the NRs, or a requirement for additional factors not present in yeast.

Studies in mammalian cells have shown that p160 proteins such as SRG1 are essential for maximal activity of $\mathrm{ER} \alpha$ both in vivo and in vitro (Sheppard et al. 2001, Li et al. 2000, Kim et al. 2001). The ability of SRG1 proteins to stimulate steroid receptor activity in yeast (Fig. 2B \& Fig. 2C) is intriguing, given the absence of close homologs of $\mathrm{CBP} / \mathrm{p} 300$ in this organism. Our results have established that the sequences containing $\mathrm{AD} 1$ and $\mathrm{AD} 2$, which function in mammalian cells by recruiting $\mathrm{CBP} / \mathrm{p} 300$ and CARM1, respectively, are also potent transactivation domains in yeast (Fig. 4A). However further mapping of the $\mathrm{AD} 1$ region revealed that the sequence required for activity (926-940; termed Helix A) is coincident with, but not identical to, the CBP recruitment domain (926-970; Helix A and Helix B) (Fig. 4B). Interestingly, a study of the sequences required for the function of the $\mathrm{N}$-terminal transactivation domain (AF1) of ER $\alpha$ also concluded that the surfaces required for AF-1 activity are not identical in yeast and mammalian cells (Metzger et al. 1995).

The structure of the CBP/ACTR interface has recently been reported and shows that the $\mathrm{AD} 1$ and SID domains each contain three $\alpha$-helices (Demarest et al. 2002). The structure confirms that Helix A makes intimate hydrophobic contacts with leucine residues within the GBP SID domain. The potent transcriptional activity of Helix $\mathrm{A}$ in yeast suggests that it mediates strong interactions with yeast nuclear proteins. Thus, short amphipathic $\alpha$-helices, such as those in the ADl and SID domains, and LXXLL motifs appear to be functionally conserved protein-protein interaction modules in eukaryotic nuclear proteins. It will be of interest to identify the yeast proteins that bind Helix A.

Our results revealed that a mutant SRCle lacking residues 901-950, which deletes most of the ADl sequence including Helix A, retained approximately $60 \%$ of the coactivator activity compared to wild type SRCle (Fig. 3G). This is in contrast to our results in mammalian cells, in which a $\triangle \mathrm{AD} 1$ mutation abrogated the ability of SRC1 proteins to enhance transactivation by $\mathrm{ER} \alpha$ (Sheppard et al. 2001), and suggests that the Helix $\mathrm{A}$ is not the major determinant required for SRC1 function in yeast. Thus, other SRG1 sequences (including amino acids 780-900) appear to be involved in contacting yeast proteins to enhance transcription of the reporter gene. The increased reporter activation achieved using SRC1 mutants deleting the $\mathrm{C}$-terminus up to amino acid 980 may indicate a repressive action of the $\mathrm{C}$-terminus of SRC1. A similar mechanism has been invoked to explain the differential potency of SRCla and SRCle isoforms in mammalian cells (Kalkhoven et al. 1998). 
We have shown previously that a truncated SRC1 protein (626-970) which contains only CBP-binding and NR-binding domains enhances the activity of $\mathrm{ER} \alpha$ to the same level as full length SRC1 proteins in reporter assays in transiently transfected COS-1 cells (Sheppard et al. 2001). The SRC1 626-970 protein is also a potent activator of $\mathrm{ER} \alpha$ in yeast, giving a 2-fold higher reporter activity than wild type SRC1 (Fig. 3B). The dispensability of bHLH-PAS and AD2 domains for SRC1 function in these assays could have several explanations: (1) it may indicate differential requirement for cofactors on different promoters; (2) additional cofactors are recruited via alternative interactions e.g. via $\mathrm{CBP} / \mathrm{p} 300$; (3) cofactor requirements are not identical for natural genes and plasmidic reporter genes, due to differences in chromatin status or (4) these domains are required for interactions with other transcription factors such as Stats, MEF2C, etc. Additionally, the relative expression levels of activators and cofactors may influence transcriptional efficacy.

In conclusion, this study and others (Metzger et al. 1995) indicate that ectopically expressed mammalian transcription factors may utilise distinct molecular mechanisms to engage the transcriptional machinery in yeast and mammalian cells. Further investigation will be required to determine the extent of the differences between these experimental models.

\section{Acknowledgements}

We are grateful to many colleagues for gifts of strains, plasmids or antibodies including Pierre Chambon, Regine Losson, Charlotte Bevan, Yoshihiro Nakatani, Eric Kalkhoven, Malcolm Parker and Tony Kouzarides. The work was supported by the Wellcome Trust.

\section{References}

Allegretto EA, McClurg MR, Lazarchik SB, Clemm DL, Kerner SA, Elgort MG, Boehm MF, White SK, Pike JW \& Heyman RA 1993 Transactivation properties of retinoic acid and retinoid $\mathrm{X}$ receptors in mammalian cells and yeast: correlation with hormone binding and effects of metabolism. Fournal of Biological Chemistry 268 26625-26633.

Anafi M, Yang YF, Barlev NA, Govindan MV, Berger SL, Butt TR \& Walfish PG 2000 GCN5 and ADA adaptor proteins regulate triiodothyronine/GRIP1 and SRC-1 coactivator-dependent gene activation by the human thyroid hormone receptor. Molecular Endocrinology 14 718-732.
Baniahmad C, Nawaz Z, Baniahmad A, Gleeson MA, Tsai MJ \& O'Malley BW 1995 Enhancement of human estrogen receptor activity by SPT6: a potential coactivator. Molecular Endocrinology 9 $34-43$.

Bevan CL, Hoare S, Claessens F, Heery DM \& Parker MG 1999 The AF1 and AF2 domains of the androgen receptor interact with distinct regions of SRC. Molecular and Cellular Biology 19 8383-8392.

Blanco JC, Minucci S, Lu J, Yang XJ, Walker KK, Chen H \& Evans RM 1998 The histone acetylase P/CAF is a nuclear receptor coactivator. Genes and Development 12 1638-1651.

Chen D, Ma H, Hong H, Koh SS, Huang SM, Schurter BT, Aswad DW \& Stallcup MR 1999 Regulation of transcription by a protein methyltransferase. Science $2842174-2177$.

Coulthard VH, Matsuda S \& Heery DM 2003 An extended LXXLL motif sequence determines the nuclear receptor-binding specificity of TRAP220. Fournal of Biological Chemistry $\mathbf{2 7 8}$ 10942-10951.

Danielian PS, White R, Lees JA \& Parker MG 1992 Identification of a conserved region required for hormone dependent transcriptional activation by steroid hormone receptors. EMBO Fournal 11 1025-1033

Demarest SJ, Martinez-Yamout M, Chung J, Chen H, Xu W, Dyson HJ, Evans RM \& Wright PE 2002 Mutual synergistic folding in recruitment of CBP/p300 by p160 nuclear receptor coactivators. Nature 415 549-553.

Gaudon C, Chambon P \& Losson R 1999 Role of the essential yeast protein PSU1 in transcriptional enhancement by the ligand-dependent activation function AF-2 of nuclear receptors. EMBO Journal 18 2229-2240.

Gilbert DM, Heery DM, Losson R, Chambon P \& Lemoine Y 1993 Estradiol-inducible squelching and cell growth arrest by a chimeric VP16-estrogen receptor expressed in Saccharomyces cerevisiae: suppression by an allele of PDR1. Molecular and Cellular Biology 13 462-472.

Guarente L \& Bermingham-McDonogh O 1992 Conservation and evolution of transcriptional mechanisms in eukaryotes. Trends in Genetics 8 27-32.

Hall BL, Smit-McBride Z \& Privalsky ML 1993 Reconstitution of retinoid $\mathrm{X}$ receptor function and combinatorial regulation of other nuclear hormone receptors in the yeast Saccharomyces cerevisiae. PNAS $906929-6933$.

Heery DM, Zacharewski T, Pierrat B, Gronemeyer H, Chambon P \& Losson R 1993 Efficient transactivation by retinoic acid receptors in yeast requires retinoid $\mathrm{X}$ receptors. PNAS $\mathbf{9 0}$ $4281-4285$.

Heery DM, Pierrat B, Gronemeyer H, Chambon P \& Losson R 1994 Homo- and heterodimers of the retinoid X receptor (RXR) activated transcription in yeast. Nucleic Acids Research 22 726-731.

Heery DM, Kalkhoven E, Hoare S \& Parker MG 1997 A signature motif in transcriptional co-activators mediates binding to nuclear receptors. Nature 387 733-736.

Heery DM, Hoare S, Hussain S, Parker MG \& Sheppard H 2001 Core LXXLL motif sequences in CREB-binding protein, SRC1, and RIP140 define affinity and selectivity for steroid and retinoid receptors. Fournal of Biological Chemistry 276 6695-6702.

Henttu PM, Kalkhoven E \& Parker MG 1997 AF-2 activity and recruitment of steroid receptor coactivator 1 to the estrogen receptor depend on a lysine residue conserved in nuclear receptors. Molecular and Cellular Biology 17 1832-1839.

Imhof MO \& McDonnell DP 1996 Yeast RSP5 and its human homolog hRPF1 potentiate hormone-dependent activation of transcription by human progesterone and glucocorticoid receptors. Molecular and Cellular Biology 16 2594-2605.

Jacq X, Brou C, Lutz Y, Davidson I, Chambon P \& Tora L 1994 Human TAFII30 is present in a distinct TFIID complex and is 
required for transcriptional activation by the estrogen receptor. Cell 79 107-117.

Kalkhoven E, Valentine JE, Heery DM \& Parker MG 1998 Isoforms of steroid receptor co-activator 1 differ in their ability to potentiate transcription by the oestrogen receptor. EMBO fournal $17232-243$

Kim MY, Hsiao SJ \& Kraus WL 2001 A role for coactivators and histone acetylation in estrogen receptor alpha-mediated transcription initiation. EMBO Fournal $206084-6094$.

Le Douarin B, Pierrat B, vom Baur E, Chambon P \& Losson R 1995 A new version of the two-hybrid assay for detection of protein-protein interactions. Nucleic Acids Research 23 876-878.

Le Douarin B, Heery DM, Gaudon C, vom Baur E \& Losson R 2001 Yeast two-hybrid screening for proteins that interact with nuclear hormone receptors. In Methods in Molecular Biology, vol 176, Steroid Receptor Methods: Protocols and Assays pp 227-248. Totowa, NJ, USA: Humana Press Inc.

Lee JW, Ryan F, Swaffield JC, Johnston SA \& Moore DD 1995 Interaction of thyroid-hormone receptor with a conserved transcriptional mediator. Nature 374 91-94.

Li J, O'Malley BW \& Wong J 2000 p300 requires its histone acetyltransferase activity and SRC-1 interaction domain to facilitate thyroid hormone receptor activation in chromatin. Molecular and Cellular Biology 20 2031-2042.

McEwan IJ 2001 Bakers yeast rises to the challenge: reconstitution of mammalian steroid receptor signalling in S. cerevisiae. Trends in Genetics 17 239-243.

Mak P, McDonnell DP, Weigel NL, Schrader WT \& O'Malley BW 1989 Expression of functional chicken oviduct progesterone receptors in yeast (Saccharomyces cerevisiae). Fournal of Biological Chemistry 264 21613-21618.

Metzger D, Whit JH \& Chambon P 1988 The human oestrogen receptor functions in yeast. Nature 334 31-36.

Metzger D, Losson R, Bornert JM, Lemoine Y \& Chambon P 1992 Promoter specificity of the two transcriptional activation functions of the human oestrogen receptor in yeast. Nucleic Acids Research 20 2813-2817.

Metzger D, Ali S, Bornert JM \& Chambon P 1995 Characterization of the amino-terminal transcriptional activation function of the human estrogen receptor in animal and yeast cells. Fournal of Biological Chemistry 270 9535-9542.

Meyer ME, Quirin-Stricker C, Lerouge T, Bocquel MT \& Gronemeyer H 1992 A limiting factor mediates the differential activation of promoters by the human progesterone receptor isoforms. Fournal of Biological Chemistry 267 10882-10887.

Needham M, Raines S, McPheat J, Stacey G, Ellston J, Hoare S and Parker M 2000 Differential interaction of steroid hormone receptors with LXXLL motifs in SRC-1 a depends on residues flanking the motif. Fournal of Steroid Biochemistry and Molecular Biology $7235-46$.

Nolte RT, Wisely GB, Westin S, Cobb JE, Lambert MH, Kurokawa R, Rosenfeld MG, Wilson TM, Glass CK \& Milburn MV 1998
Ligand binding and co-activator assembly of the peroxisome proliferator-activated receptor-gamma. Nature 395 137-143.

Onate SA, Tsai SY, Tsai MJ \& O’Malley BW 1995 Sequence and characterization of a coactivator for the steroid hormone receptor superfamily. Science 270 1354-1357.

Picard D, Khursheed B, Garabedian MJ, Fortin MG, Lindquist S \& Yamamoto KR 1990 Reduced levels of hsp90 compromise steroid receptor action in vivo. Nature 348 166-168.

Pierrat B, Heery DM, Lemoine Y \& Losson R 1992 Functional analysis of the human estrogen receptor using a phenotypic transactivation assay in yeast. Gene 119 237-245.

Pierrat B, Heery DM, Chambon P \& Losson R 1994 A highly conserved region in the hormone-binding domain of the human estrogen receptor functions as an efficient transactivation domain in yeast. Gene $\mathbf{1 4 3} 193-200$.

Sheppard HM, Harries JC, Hussain S, Bevan C \& Heery DM 2001 Analysis of the steroid receptor coactivator 1 (SRC1)-CREB binding protein interaction interface and its importance for the function of SRC1. Molecular and Cellular Biology 21 39-50.

Shiau AK, Barstad D, Loria PM, Cheng L, Kushner PJ, Agard DA \& Greene GL 1998 The structural basis of estrogen receptor/coactivator recognition and the antagonism of this interaction by tamoxifen. Cell $\mathbf{9 5} 927-937$.

vom Baur E, Zechel C, Heery D, Heine MJ, Garnier JM, Vivat V, Le Douarin B, Gronemeyer H, Chambon P \& Losson R 1996 Differential ligand-dependent interactions between the AF-2 activating domain of nuclear receptors and the putative transcriptional intermediary factors mSUG1 and TIF1. EMBO fournal 15 110-124.

vom Baur E, Harbers M, Um SJ, Benecke A, Chambon P \& Losson R 1998 The yeast Ada complex mediates the ligand-dependent activation function AF-2 of retinoid $\mathrm{X}$ and estrogen receptors. Genes and Development 12 1278-1289.

Wallberg AE, Neely KE, Hassan AH, Gustafsson JA, Workman JL \& Wright AP 2000 Recruitment of the SWI-SNF chromatin remodeling complex as a mechanism of gene activation by the glucocorticoid receptor taul activation domain. Molecular and Cellular Biology $202004-2013$

White R, Sjoberg M, Kalkhoven E \& Parker MG 1997 Ligand-independent activation of the oestrogen receptor by mutation of a conserved tyrosine. EMBO fournal 16 1427-1435.

Yang XJ, Ogryzko VV, Nishikawa J, Howard BH, Nakatani Y 1996 A p300/CBP-associated factor that competes with the adenoviral oncoprotein E1A. Nature 382 319-324.

Yoshinaga SK, Peterson CL, Herskowitz I \& Yamamoto KR 1992 Roles of SWI1, SWI2, and SWI3 proteins for transcriptional enhancement by steroid receptors. Science 258 1598-1604.

\section{Received in final form 10 February 2003} Accepted 24 February 2003 